\title{
Diamagnetic coupling for magnetic tuning in nano-thin films
}

Cite as: Appl. Phys. Lett. 116, 252402 (2020); https://doi.org/10.1063/5.0008482

Submitted: 22 March 2020. Accepted: 04 June 2020 . Published Online: 23 June 2020

M. M. Vopson (iD), M. Belusky (iD, and S. Lepadatu (D)

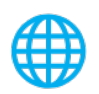

\section{ARTICLES YOU MAY BE INTERESTED IN}

Optoelectronic domain-wall motion for logic computing

Applied Physics Letters 116, 252403 (2020); https://doi.org/10.1063/5.0013369

Thermally induced generation and annihilation of magnetic chiral skyrmion bubbles and achiral bubbles in $\mathrm{Mn}-\mathrm{Ni}-\mathrm{Ga}$ magnets

Applied Physics Letters 116, 132402 (2020); https://doi.org/10.1063/1.5142083

Direct measurement of interfacial Dzyaloshinskii-Moriya interaction at the $\mathrm{MoS}_{2} / \mathrm{Ni}_{80} \mathrm{Fe}_{20}$ interface

Applied Physics Letters 116, 232405 (2020); https://doi.org/10.1063/5.0009828

\section{Lock-in Amplifiers up to $600 \mathrm{MHz}$}
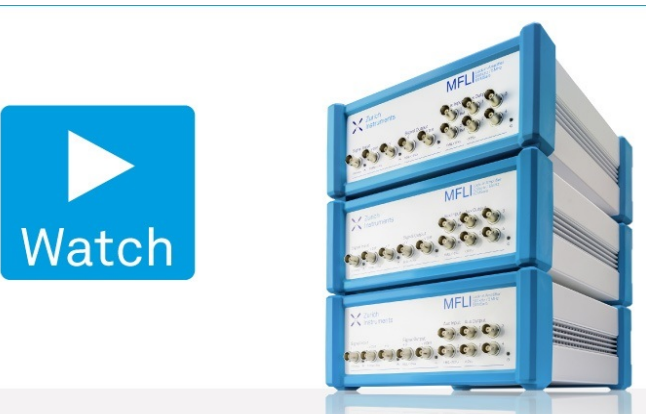


\title{
Diamagnetic coupling for magnetic tuning in nano-thin films
}

\author{
Cite as: Appl. Phys. Lett. 116, 252402 (2020); doi: 10.1063/5.0008482 \\ Submitted: 22 March 2020 - Accepted: 4 June 2020 . \\ Published Online: 23 June 2020

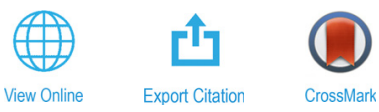

M. M. Vopson, ${ }^{1, a)}$ (D) M. Belusky, (iD and S. Lepadatu² (D)

\begin{abstract}
AFFILIATIONS
'School of Mathematics and Physics, University of Portsmouth, Portsmouth PO1 3QL, United Kingdom

2 Jeremiah Horrocks Institute for Mathematics, Physics and Astronomy, University of Central Lancashire, Preston PR1 2HE, United Kingdom
\end{abstract}

a) Author to whom correspondence should be addressed: melvin.vopson@port.ac.uk; formerly known as Vopsaroiu.

\begin{abstract}
Combining nanoscale thin films of magnetic and non-magnetic phases in various hetero-structures has generated a rich variety of new magnetic and magneto-transport phenomena and applications. Here, we propose a coupling between ferromagnetic and diamagnetic layers. We used this diamagnetic coupling to improve the exchange bias field of a diamagnet/ferromagnet/anti-ferromagnet hetero-structure by up to $212 \%$, as evidenced in the experiments presented here. Since diamagnets have very special properties, including temperature independent negative magnetic susceptibilities, this coupling could be a powerful tool in future synthesis of solid state nanostructures such as exchange bias systems, spintronic devices, magnetic random access memories, sensors, and multiferroics.
\end{abstract}

Published under license by AIP Publishing. https://doi.org/10.1063/5.0008482

The exchange bias effect defined as the field shift in the magnetic hysteresis loop of a ferromagnetic layer, ${ }^{1-3}$ is an essential component of advanced sensors, spintronic devices, existing magnetic data storage, and future magnetic memories. Depositing an anti-ferromagnetic layer in intimate contact with a ferromagnetic layer induces the exchange bias effect, which arises from the quantum mechanical exchange coupling between adjacent spins at the ferromagnetic/anti-ferromagnetic interface [Fig. 1(a)].

Although the effect is fully understood, much research is currently dedicated to finding new ways to enhance and manipulate the exchange bias field. ${ }^{4-}$

Here, we show that diamagnetic coupling is a promising approach to fabricating magnetic hetero-structures and multilayers that can be used to significantly enhance the exchange bias effect. By using a diamagnetic underlayer for exchange bias structures, we were able to demonstrate that the negative magnetic susceptibility of the diamagnet reduces the effective positive magnetic susceptibility of the magnetic layer, enhancing significantly the overall exchange bias effect.

Diamagnetic materials have recently attracted increasing interest due to their electronic surface-state spin and valley properties ${ }^{8}$ and the discovery of the oscillatory magneto-caloric effect in diamagnetic solids. ${ }^{9}$ Our work further demonstrates the potential of diamagnetic materials, by introducing diamagnetic layers in complex magnetic hetero-structures to achieve manipulation of magnetic properties, which may lead to future innovative spintronic designs incorporating diamagnets.

The exchange bias field given by the Meiklejohn-Bean formula ${ }^{10}$ shows that the strength of the exchange bias field is inversely proportional to the magnetic moment of the ferromagnetic layer per surface area, usually represented as the product of its magnetization and thickness

$$
H_{e x} \approx \frac{J}{M_{F} t_{F}},
$$

where $\mathrm{H}_{\mathrm{ex}}$ is the exchange bias field, $\mathrm{J}$ is the exchange coupling constant between anti-ferromagnetic (AF) and ferromagnetic (F) spins at

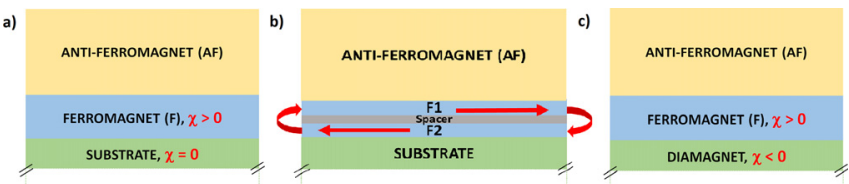

FIG. 1. (a) Schematic diagram of the standard exchange bias structure on an ordinary substrate, $\chi=0$; (b) exchange bias structure with a synthetic anti-ferromagnet (SAF); and (c) identical structure to (a) fabricated onto a diamagnetic substrate, $\chi<0$. 
the interface, and $\mathrm{M}_{\mathrm{F}}$ and $\mathrm{t}_{\mathrm{F}}$ are the magnetization and thickness, respectively, of the ferromagnetic layer. For this reason, very thin ferromagnetic layers $\left(t_{F}\right.$ is typically $4-10 \mathrm{~nm}$ ) of moderate $M_{F}$ values, such as NiFe permalloy, are frequently utilized to maximize the exchange bias field. Reducing the thickness $t_{F}$, or choosing materials of low $\mathrm{M}_{\mathrm{F}}$, are effective ways of boosting the exchange bias field. However, too thin magnetic layers, or very low $M_{F}$ values are not desirable as they reduce the functionality and sensitivity of the overall device. Another way of enhancing the exchange bias field is to minimize $M_{F}$ by replacing the pinned ferromagnetic layer with a pinned tri-layer structure called synthetic anti-ferromagnet (SAF), ${ }^{11}$ consisting of two ferromagnetic layers separated by a non-magnetic spacer [Fig. 1(b)]. Such systems display an interlayer exchange coupling between the two ferromagnetic layers adjacent to the same non-magnetic layer. $^{12,13}$ The coupling is known as Ruderman-Kittel-Kasuya-Yosida (RKKY) coupling and it leads to an oscillatory interlayer exchangecoupling constant that oscillates with the distance between the ferromagnetic layers. ${ }^{14-16}$ When the spacer is too thick, typically thicker than $40 \mathrm{~nm}$, the interlayer exchange coupling is suppressed. However, for a very thin non-magnetic spacer layer, by changing its thickness, the interlayer exchange coupling interaction can be tuned from ferromagnetic (magnetization of the ferromagnetic layers in parallel alignment) to anti-ferromagnetic (ferromagnetic layers in an anti-parallel alignment), also known as synthetic anti-ferromagnet (SAF).

Exchange bias systems with an SAF pinned layer [see Fig. 1(b)] display an effective magnetic moment that participates in the exchange bias, $\mathrm{m}_{\mathrm{eff}}$, which is the vector sum of the magnetic moment vectors within the SAF structure, written in absolute values as

$$
m_{\text {eff }}=m_{F 1}-m_{F 2} .
$$

Recalling that the magnetization is the magnetic moment per unit volume, and introducing the relation between magnetization and applied field, $\mathrm{M}=\chi \cdot \mathrm{H}$, relation (2) becomes

$$
\chi_{e f f}=\chi_{F 1}-\chi_{F 2} \cdot \frac{t_{F 2}}{t_{F 1}},
$$

where: $\chi_{F 1}, \chi_{F 2}$ are the magnetic susceptibilities of the magnetic $F_{1}$ and $\mathrm{F}_{2}$ layer, respectively, $t_{F 1}, t_{F 2}$ are their corresponding thickness, and $\chi_{\text {eff }}$ is the effective magnetic susceptibility of the SAF.

By choosing ferromagnetic SAF layers of identical composition $\left(\chi_{F 1}=\chi_{F 2}\right)$ and thickness $\left(t_{F 1}=t_{F 2}\right)$, the effective magnetic susceptibility of the SAF structure is $\chi_{\text {eff }} \approx 0$, resulting in almost zero effective magnetization/magnetic moment participating in the exchange bias effect, so according to (1), $\mathrm{H}_{\mathrm{ex}}$ is maximized.

Here, we propose a different mechanism that achieves a similar result without using an SAF structure. In our proposed concept, the coupling to a diamagnetic material of negative magnetic susceptibility lowers the effective magnetic moment participating in the exchange bias effect. To understand this effect, let us assume that an exchange bias hetero-structure is fabricated as shown in Fig. 1(a), onto a standard substrate such as Si. Let us denote $\mathrm{m}_{\mathrm{F}}$ as the magnetic moment of the ferromagnetic layer. The same structure is then fabricated simultaneously onto a diamagnetic underlayer as seen in Fig. 1(c). The most cost-effective material available with the best diamagnetic susceptibility is Bismuth (Bi). By growing the exchange bias structure onto diamagnetic $\mathrm{Bi}$, the structure could be seen as an exchange bias structure with an effective magnetic moment given by

$$
m_{\text {eff }}=m_{F}-m_{\text {dia }} .
$$

Relation (4) is written in absolute values for positive applied magnetic fields. The same relation is valid for negative applied magnetic fields, except that for negative magnetic fields, relation (4) is multiplied by $(-1)$ on both sides. This is very similar to the effective magnetic moment of the SAF structure, but in this case, the magnetic moment reduction results from the negative contribution of the diamagnetic underlayer, instead of the anti-parallel interlayer exchange coupling, as in the SAF. Introducing the magnetic susceptibility of the magnetic $\mathrm{F}$ layer, $\chi_{F}$, the diamagnetic susceptibility of the underlayer, $\chi_{\text {dia }}$, and their corresponding thicknesses $t_{F}, t_{d i a}$, the effective magnetic susceptibility of the diamagnetic exchanged biased structure, $\chi_{\text {eff }}$ is:

$$
\chi_{\text {eff }}=\chi_{F}-\chi_{\text {dia }} \cdot \frac{t_{\text {dia }}}{t_{F}},
$$

where $\chi_{\text {dia }}$ in (5) is the absolute value. Since the two-coupled materials are magnetically very different, unlike the SAF where the condition $\left(t_{F 1}=t_{F 2}\right)$ is sufficient to produce an effective susceptibility of almost zero value, the cancelation of the effective susceptibility, $\chi_{\text {eff }}=0$ in (5), imposes a strict relationship between the thickness of the ferromagnetic-pinned layer and the diamagnetic underlayer

$$
t_{\text {dia }}=\frac{\chi_{F}}{\chi_{\text {dia }}} \cdot t_{F}
$$

A typical ferromagnetic material used in exchange bias systems is $\mathrm{NiFe}$ permalloy, of $7 \mathrm{~nm}$ thickness and magnetic susceptibility $\chi_{F}=\chi_{\text {NiFe }}=8 \times 10^{3.17}$ The best material choice in terms of largest diamagnetic susceptibility is $\mathrm{Bi}, \chi_{\text {dia }}=\chi_{B i}=-16.6 \times 10^{-5.18}$ Using these numerical values in (6), we obtain the thickness of the diamagnetic underlayer required to produce a zero effective magnetic moment/ effective susceptibility, $t_{\text {dia }}=0.25 \mathrm{~m}$. This thickness is very large, but most likely overestimated because the ferromagnet permalloy saturates at a relatively low magnetic field, while the diamagnet increases its magnetization linearly with the applied field, indefinite, without saturation. For a $7 \mathrm{~nm}$ soft ferromagnetic layer, saturating at 30 Oe magnetic field, we estimate that the required thickness of the $\mathrm{Bi}$ diamagnetic underlayer in order to achieve the total effective magnetic moment cancelation at $1000 \mathrm{Oe}$, is $t_{d i a}=7.3 \times 10^{-3} \mathrm{~m}$. This thickness is still rather large, but the same coupling could be induced with much thinner diamagnetic layers, without achieving total cancelation of the effective magnetization. In this study, we predict that the coupling of a nano-scale ferromagnet to a much larger diamagnetic underlayer would result in a significant reduction of the effective magnetization of the overall structure, which in turn could be used to tune the exchange bias effect, or other magnetic properties of the overall hetero-structure.

In order to test our concept of diamagnetic tuning of the exchange bias field, we fabricated samples using a KJL LabLine 5 magnetron plasma sputtering system. ${ }^{19}$ The $5 \times 5 \mathrm{~mm}$ size samples had a vertical structure: $\mathrm{Si} / \mathrm{Bi}(\mathrm{t}) / \mathrm{NiFe}(7 \mathrm{~nm}) / \mathrm{IrMn}(15 \mathrm{~nm})$. Five samples with this structure have been sputtered under identical conditions. One was a control sample consisting of a standard $S i$ wafer substrate with $t_{B i}=0 \mathrm{~nm}$, and the other four samples were coated onto diamagnetic Bi underlayers of $\mathrm{t}_{\mathrm{Bi}}=100 \mathrm{~nm}, 300 \mathrm{~nm}, 600 \mathrm{~nm}$, and $900 \mathrm{~nm}$ thickness, respectively.

The base pressure was $1.5 \times 10^{-7}$ Torr, the argon process pressure was kept constant at $3 \times 10^{-3}$ Torr, and the sputtering gun was kept at $60 \mathrm{~W}$ DC power. These resulted in average sputtering rates of $2 \AA / s$ for 
$\mathrm{NiFe}$ and $1.5 \AA / \mathrm{s}$ for IrMn. The deposition of Bi required a reduction of the DC power down to $20 \mathrm{~W}$, as Bi has a low melting point. Magnetic hysteresis loops were measured at room temperature using an in-house developed Magneto-Optic Kerr Effect (MOKE) system. Figure 2 shows the magnetic hysteresis loops of each sample, from which we extracted the exchange bias values. Samples sputtered onto the Si substrate without the Bi underlayer display an exchange bias field of 54 Oe. Samples coated on $\mathrm{Bi}$, display an increased exchange bias field, as predicted by our proposed coupling concept. The $100 \mathrm{~nm}, 300 \mathrm{~nm}, 600 \mathrm{~nm}$, and $900 \mathrm{~nm}$ Bi samples have an exchange bias field of 119 Oe, 159 Oe, 166 Oe, and 169 Oe, respectively.

These correspond to increases of $120 \%, 194 \%, 207 \%$, and $212 \%$ in the exchange bias field due to the diamagnetic coupling, respectively.

The thicker the diamagnet, the larger is the effect observed. However, it appears that the effect displays saturation and the differences observed when increasing the Bi thickness from $300 \mathrm{~nm}$ to $600 \mathrm{~nm}$ are very small. To emphasize the observed coupling effect, we show in Fig. 3 the exchange bias field values as a function of the diamagnetic Bi thickness. Extended studies of roughness effects in NiFe thin films ${ }^{20}$ undertaken in our laboratory, combined with identical experiments to those reported in this article, but using very thick Bi substrates with rough surfaces, allowed us to rule out the possibility that our results could be explained by structural or interfacial roughness effects. Since our initial calculations indicate that a much thicker Bi underlayer should induce these large reductions in the effective magnetic moment (i.e., large increases in the exchange bias field), we conclude that the function of the diamagnetic underlayer in this coupling effect is not just to reduce the effective magnetic moment, but also to possibly induce a subtler interfacial magnetic coupling, which requires further investigations.

Although not fully understood, our results indicate that there could be some kind of interfacial coupling between ferromagnets and

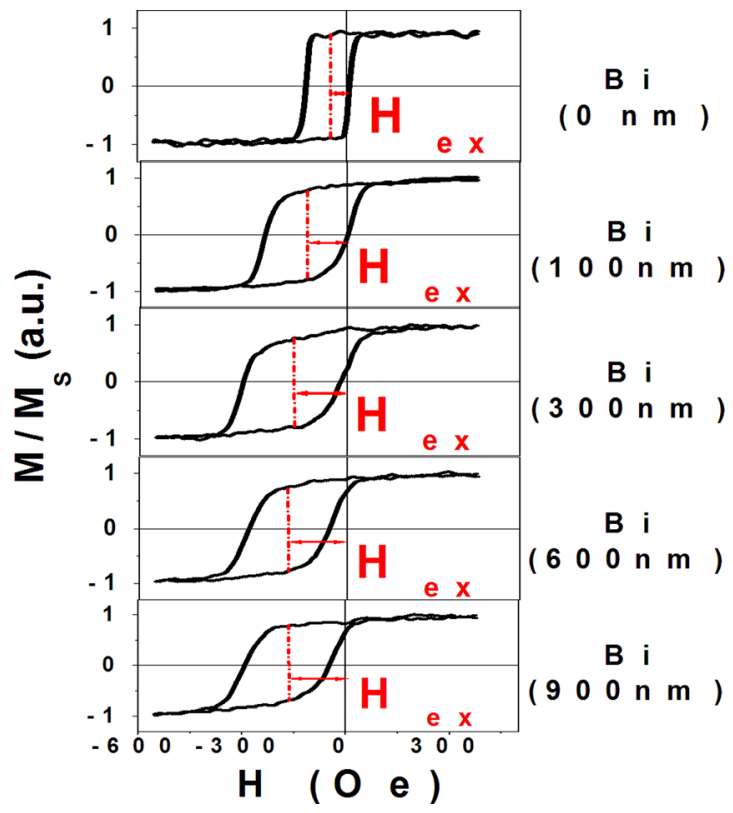

FIG. 2. Magnetic hysteresis loops at room temperature measured via MOKE. From top down: $\mathrm{Bi}=0 \mathrm{~nm} ; \mathrm{Bi}=100 \mathrm{~nm} ; \mathrm{Bi}=300 \mathrm{~nm} ; \mathrm{Bi}=600 \mathrm{~nm}$, and $\mathrm{Bi}=900 \mathrm{~nm}$.

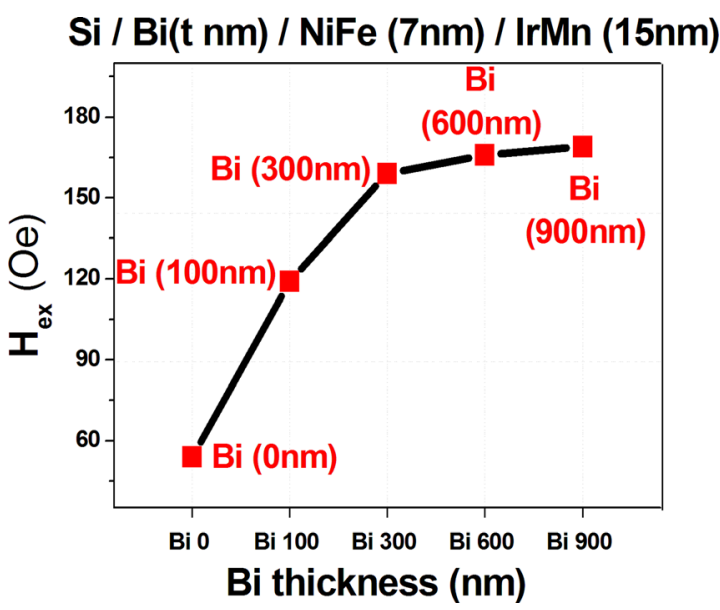

FIG. 3. Exchange bias field as a function of the thickness of the diamagnetic Bi underlayer. The coupling of the negative susceptibility diamagnetic $\mathrm{Bi}$ to the NiFe, significantly increases the exchange bias field.

diamagnets at the nano-scale. The observed dependence on the $\mathrm{Bi}$ layer thickness suggests that, if such interfacial coupling exists, the surface state of $\mathrm{Bi}$ is strongly influenced by the bulk, possibly leading to a variation of the coupling strength. We therefore hope that this article will stimulate further experimental and theoretical work in order to fully understand the exact origin of this possible coupling term.

We conclude that the diamagnetic coupling is a convenient way of tuning magnetism. In this study, we demonstrated tuning of the exchange bias effect via diamagnetic coupling. However, it is expected that diamagnetic coupling to ferromagnetic layers and the complex hetero-structure will become a synthesis and control tool not only in future exchange bias systems, but also spintronic devices, ${ }^{21}$ magnetic random access memories, ${ }^{22}$ sensors, and multiferroic structures. ${ }^{23}$ Moreover, the fact that diamagnetic materials have a negative susceptibility, combined with the fact that their susceptibility is temperature independent, makes the diamagnetic coupling a tool for applications where thermal and caloric effects are of interest, as the coupling is most likely unaffected by the temperature. This feature is unique and makes diamagnetic coupling very different from all other coupling mechanisms, including RKKY, ${ }^{14-16}$ Dzyaloshinskii-Moriya coupling, ${ }^{24,25}$ which are all strongly temperature dependent.

\section{AUTHORS' CONTRIBUTIONS}

All authors contributed equally to this work.

The authors gratefully acknowledge the financial support for this work received from Kurt J. Lesker Ltd and the University of Portsmouth via a Ph.D. studentship funding.

\section{DATA AVAILABILITY}

The data that support the findings of this study are available from the corresponding author upon reasonable request.

\section{REFERENCES}

1J. Nogués and I. K. Schuller, “Exchange bias," J. Magn. Magn. Mater. 192, 203-232 (1999).

${ }^{2}$ M. Kiwi, "Exchange bias theory,” J. Magn. Magn. Mater. 234, 584-595 (2001). 
${ }^{3}$ K. O'Grady, L. E. Fernandez-Outon, and G. Vallejo-Fernandez, "A new paradigm for exchange bias in polycrystalline thin films," J. Magn. Magn. Mater. 322, 883-899 (2010).

${ }^{4}$ P.-H. Lin, B.-Y. Yang, M.-H. Tsai, P.-C. Chen, K.-F. Huang, H.-H. Lin, and C.H. Lai, "Manipulating exchange bias by spin-orbit torque," Nat. Mater. 18, 335-341 (2019).

${ }^{5} \mathrm{Q}$. Yang, Z. Zhou, N. X. Sun, and M. Liu, "Perspectives of voltage control for magnetic exchange bias in multiferroic heterostructures," Phys. Lett. A 381(14), 1213-1222 (2017).

${ }^{6}$ S. Harisankar, M. Chandra, and K. R. Mavani, "Tuning of exchange bias with interfacial ferromagnetism in $\mathrm{NdNiO}_{3} / \mathrm{NdMnO}_{3}$ heterostructures," J. Magn. Magn. Mater. 477(1), 35-41 (2019).

${ }^{7}$ W. Yuan, T. Su, Q. Song, X. Wenyu, C. Yangyang, W. Tianyu, Z. Zhangyuan, M. Xiumei, G. Peng, S. Jing, and H. Wei, "Crystal structure manipulation of the exchange bias in an antiferromagnetic film," Sci. Rep. 6, 28397 (2016).

${ }^{8}$ H. Du, X. Sun, X. Liu, X. Wu, J. Wang, M. Tian, A. Zhao, Y. Luo, J. Yang, B. Wang, and J. G. Hou, "Surface Landau levels and spin states in bismuth (111) ultrathin films," Nat. Commun. 7, 10814 (2016).

${ }^{9}$ M. S. Reis, "Oscillating magnetocaloric effect," Appl. Phys. Lett. 99, 052511 (2011).

${ }^{10}$ W. H. Meiklejohn and C. P. Bean, Phys. Rev. 102, 1413 (1956).

"J. G. Zhu, "Spin valve and dual spin valve heads with synthetic antiferromagnets," IEEE Trans. Magn. 35, 655-660 (1999).

${ }^{12}$ P. Grunberg, R. Schreiber, Y. Pang, M. B. Brodsky, and H. Sowers, "Layered magnetic structures: Evidence for antiferromagnetic coupling of Fe layers across Cr interlayers," Phys. Rev. Lett. 57, 2442-2445 (1986).

${ }^{13}$ C. F. Majkrzak, J. W. Cable, J. Kwo, M. Hong, D. B. McWhan, Y. Yafet, J. V. Waszczak, and C. Vettier, "Observation of a magnetic antiphase domain structure with long-range order in a synthetic Gd-Y superlattice," Phys. Rev. Lett. 56, 2700-2703 (1986).
${ }^{14} \mathrm{~J}$. C. Slonczewski, "Conductance and exchange coupling of two ferromagnets separated by a tunneling barrier," Phys. Rev. B 39, 6995-7002 (1989).

${ }^{15}$ S. Parkin, N. More, and K. P. Roche, "Oscillations in exchange coupling and magnetoresistance in metallic superlattice structures: $\mathrm{Co} / \mathrm{Ru}, \mathrm{Co} / \mathrm{Cr}$, and $\mathrm{Fe} /$ Cr," Phys. Rev. Lett. 64, 2304 (1990).

${ }^{16} \mathrm{P}$. Bruno, "Theory of interlayer magnetic coupling," Phys. Rev. B 52, 411 (1995).

${ }^{17}$ D. Jiles, Introduction to Magnetism and Magnetic Materials (CRC Press, 1998), p. 354, ISBN: 978-0-412-79860-3.

${ }^{18} \mathrm{~S}$. Otake, M. Momiuchi, and N. Matsuno, "Temperature dependence of the magnetic susceptibility of bismuth," J. Phys. Soc. Jpn. 49(5), 1824-1828 (1980).

${ }^{19} \mathrm{~K}$. J. Lesker, see https://www.lesker.com/newweb/ped/physical-vapor-deposition-systems.cfm for "Company | Physical Vapor Deposition Systems | Vacuum Science Is Our Business."

${ }^{20}$ M. Belusky, S. Lepadatu, J. Naylor, and M. M. Vopson, "Evidence of substrate roughness surface induced magnetic anisotropy in $\mathrm{Ni}_{80} \mathrm{Fe}_{20}$ flexible thin films," J. Magn. Magn. Mater. 478, 77-83 (2019).

${ }^{21}$ A. Hirohata and K. Takanashi, "Future perspectives for spintronic devices," J. Phys. D: Appl. Phys. 47, 193001 (2014).

${ }^{22}$ B. Sabpreet, S. Rachid, H. Atsufumi, O. Hideo, F. Shunsuke, and S. N. Piramanayagam, "Spintronics based random access memory: A review," Mater. Today 20(9), 530-548 (2017).

${ }^{23} \mathrm{M}$. M. Vopson, "Fundamentals of multiferroic materials and their possible applications," Crit. Rev. Solid State Mater. Sci. 40, 223-250 (2015).

${ }^{24} \mathrm{~T}$. Moriya, "Anisotropic superexchange interaction and weak ferromagnetism," Phys. Rev. 120(1), 91 (1960).

${ }^{25}$ I. Dzyaloshinskii, "A thermodynamic theory of 'weak' ferromagnetism of antiferromagnetics," J. Phys. Chem. Solids 4(4), 241 (1958). 